\title{
DIVERSIDAD FLORÍSTICA ARBÓREA Y SU RELACIÓN CON EL SUELO EN UN BOSQUE PLUVIAL TROPICAL DEL CHOCÓ BIOGEOGRÁFICO ${ }^{1}$
}

\author{
Harley Quinto Mosquera² e Flavio Moreno Hurtado ${ }^{3}$
}

\begin{abstract}
RESUMO - El objetivo del presente estudio fue determinar la composición y diversidad florística arbórea a escala local en un bosque del Chocó biogeográfico, una de las regiones más lluviosas del mundo, y su relación con las características del sitio. Se emplearon dos parcelas de una hectárea, en las cuales se determinó la composición y diversidad de árboles con DAP $>10 \mathrm{~cm}$ y se relacionaron con los nutrientes del suelo, la topografíay el espacio. No se presentaron diferencias significativas entre las parcelas para las variables estructurales y de diversidad estudiadas, con valores de abundancia de 640 y 716 individuos/ha yde riqueza de 174 y 223 especies/ ha.Nuestros resultados soportan la hipótesis de hiperdominancia de unos pocos grupos biológicos en los bosques húmedos tropicales. Ladiversidad arbórea a escala local se relacionó significativamente con algunas condiciones edáficas.Se presentó asociación positiva de la diversidad con el aluminio del suelo, y negativa con la topografía.No obstante, solamente un 37\% de la variación en la composición florística fue explicada por las variables espaciales y ambientales (topográficas y edáficas). Por ello, aparentemente la distribución de las especies a escala local parece estar gobernada principalmente por procesos aleatorios o biológicos (como la limitación en dispersión), conforme lo propone la teoría neutral. El papel de las adaptaciones específicas al hábitat (teoría del nicho) pareciera ser limitado.
\end{abstract}

Palabras clave: Dominancia florística, teoría neutral, teoría del nicho ecológico.

\section{DIVERSIDADE FLORÍSTICA ARBÓREA E SUA RELAÇÃO COM O SOLO EM UMA FLORESTA TROPICAL NO CHOCÓ BIOGEOGRÁFICO}

\begin{abstract}
RESUMO - O objetivo deste estudo foi determinar a composição e diversidade florística arbórea localmente na floresta tropical de Chocó biogeográfico, uma das regiões mais úmidas do mundo, e sua relação com os recursos locais. Foram uUtilizadaram-se duas parcelas de um 1 hectareha, nos nas quais foram determinadas a composição e diversidade de árvores com DAP> $10 \mathrm{~cm}$ e foram avaliadas suas relações com os nutrientes do solo, a topografia e o espaço. Não houve diferenças significativas entre as parcelas para com relação àas variáveis estruturais e de diversidade estudadas, com valores de abundância de 640 e 716 indivíduos/ ha e riqueza de 174 e 223 espécies/ha. Nossos Os resultados apoiam a hipótese de hiperdominância de poucos grupos biológicos em florestas tropicais. A diversidade de árvores em escala local foi relacionada significativamente com algumas condições do solo. A associação positiva da diversidade com alumínio do solo e negativa com a topografia foi apresentada. No entanto, apenas 37\% da variação na composição de espécies foi explicada por variáveis espaciais e ambientais (topografia e solo). Então, aparentemente a distribuição das espécies em escala local parece ser governada, principalmente, por processos aleatórios ou biológicos (limitação de dispersão), conforme proposto pela teoria neutra. O papel das adaptações específicas ao hábitat (teoria de nicho) parece ser limitado.
\end{abstract}

Palavras clave: Dominância florística; Teoria neutra; Teoria de nicho.

\footnotetext{
${ }^{1}$ Recebido em 05.05.2014 aceito para publicação em 02.10.2014.

${ }^{2}$ Programa de Biología. Facultad de Ciencias Básicas, Universidad Tecnológica del Chocó "Diego Luis Córdoba, Quibdó, Colombia. E-mail: <hquintom@gmail.com>.

${ }^{3}$ Departamento de Ciencias Forestales, Facultad de Ciencias Agrarias, Universidad Nacional de Colombia sede Medellín, Medellín, Colombia.E-mail: <fhmoreno@unal.edu.co>.
} 


\section{INTRODUCCIÓN}

Los bosques tropicales están entre los ecosistemas terrestres más importantes del planeta debido a su extensión geográfica, complejidad ecológica, biodiversidad y endemismo (HARTSHORN, 2002). Albergan cerca de $37 \%$ de las H”90.000 especies de plantas fanerógamas del mundo (ANTONELLI; SANMARTIN, 2011) y pueden llegar a contener más de 473 especies de árboles y lianas en una hectárea (GENTRY, 1988; PHILLIPS et al.1994).

Algunos estudios indican que la diversidad y composiciónflorística de los bosques tropicalesson explicadas en gran medida por el clima, el drenaje, la topografía y los suelos (DUIVENVOORDEN, 1995; JOHN et al., 2007; POULSEN et al., 2006; TER STEEGE et al.,1993); tales resultados parecen apoyar la teoría del nicho, que asume que las especies coexisten como respuesta a adaptaciones específicas del hábitat, las cuales otorgan una ventaja competitiva a unas especies frente a otras (HUTCHINSON, 1959;TILMAN; PACALA, 1993). Sin embargo, otros estudios realizados a escala local en bosques similares, han encontrado que el efecto de los suelos sobre la diversidad y la variabilidad florística es mínimo, y que la mayor parte de la explicación de tal variación se presenta por procesos aleatorios o biológicos como la limitación en dispersión (DUQUEet al.,2003;HEet al., 2002; VALENCIAet al., 2004), lo cual ha recibido el nombre de teoría neutral (HUBBELL, 2001;HE; HU,2005).

El Chocó Biogeográfico es una región del neotrópicoubicada al noroeste de Sudaméricaque alberga bosques muy húmedos y pluviales tropicales con características ecológicas y biogeográficas únicas,pues presentagran variabilidad geomorfológica, ecosistémica y florística (MALAGÓN et al., 1995; RANGEL, 2004). Además, es consideradounhotspot de conservación debido a su alta biodiversidad y endemismo (MYERS et al., 2000).Allí se han registrado entre 200 y 300 especies arbóreaspor hectárea (GENTRY, 1988,1993b; FABERLANGENDOEN; GENTRY, 1991). Gentry (1988) atribuyeesta inusual diversidad a los altos niveles de precipitación y ausencia de sequías, aunqueeste supuesto realmente no ha sido contrastado, pues en esta región se han realizado pocos estudios.

El objeto del presente estudio es evaluar la composición y diversidad florística arbórea,así como la distribución de especies de un bosque pluvial tropical del Chocó y su asociación con factores espaciales y edáficos, como nutrientes del suelo y topografía. Específicamente sebusca responder los siguientes interrogantes: ¿Cómo están distribuidas las abundancias de las especies arbóreas en un bosque pluvial tropical del Chocó? ¿Existe relación entre la composición florística,los patrones de riqueza de especies, diversidad y dominancia florística arbórea con las condiciones del sitio en un bosque pluvial tropicaldel Chocó?

\section{MATERIALES Y MÉTODOS}

El presente estudio se realizó en dos parcelas permanentesinstaladas en un bosque primario en el corregimiento de Salero, municipio de Unión Panamericana (departamento del Chocó, Colombia; 5¹8' $\mathrm{N}-76^{\circ} 36^{\prime} \mathrm{W}$ ). De acuerdo con nuestros registros, laprecipitación promedia anual es de7600 mm, temperatura entre $26^{\circ} \mathrm{C}$ y $30^{\circ} \mathrm{C}$ y humedad relativa de $90 \%$; la zona de vida es bosque pluvial tropical.Los suelosson bien drenados, moderadamente superficiales, fuertemente ácidos, de baja fertilidad natural y pertenecen a los órdenesEntisoles e Inceptisoles (MALAGÓN et al., 1995).

Lasdos parcelas(denominadas E y U) distan 300 $m$ una de otra y fueron establecidas entre abril y agosto de 1998; cada una tiene $20 \mathrm{~m}$ de ancho x $500 \mathrm{~m}$ de largo ( 1 ha), dividida en 25 unidades de muestreo de $20 \mathrm{x}$ $20 \mathrm{~m}\left(400 \mathrm{~m}^{2}\right)$. Se midió el diámetro a la altura del pecho (DAP) en cm (1.30 m sobre el suelo) con cinta diamétricaa los árboles con DAP e” 10 cm. Se registró la ubicación espacial de cada individuoy su hábito de crecimiento (árbol, bejuco o liana y palma) yse tomaron muestras para identificación botánica. También se tomó unamuestra compuesta de suelo a $20 \mathrm{~cm}$ de profundidadpor unidad de muestreo para análisis químicos (50 muestras en total). Las variaciones en altitud cada $20 \mathrm{~m}$ se determinaron a partir de los datos de pendiente, la cual se midió cada 10 m con clinómetro Suunto ${ }^{\circledR}$.

Se identificaron todos los individuos hasta el máximo nivel taxonómico posible en el herbario de la Universidad Tecnológica del Chocó D.L.C. “Herbario Chocó” con las claves de Gentry (1993a) y Mahecha (1997). En las muestras de suelo se analizó pH y contenidos de materia orgánica (M.O.) y nutrientes (Ca, Mg, K y P).

Con los datos delos inventariosde árboles se estimaron los siguientes índices de diversidad y

Revista Árvore, Viçosa-MG, v.38, n.6, p.1123-1132, 2014

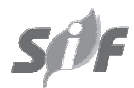


dominancia: riqueza de especies, densidad de especies de Margalef, equidad de Shannon-Wiener, coeficiente alfa de Fisher,dominancia de Simpson y de BergerParker(MAGURRAN, 1988).La comparación de los valores de diversidad entre parcelas se hizo mediante la prueba no paramétrica de Mann-Whitney-Wilcoxon, dado que no cumplieron los supuestos de normalidad y homogeneidad de varianzas, evaluados con los estadísticos de Bartlett y Hartley.

Lasrelaciones linealesindividuales entre las variables de diversidad y de sitio (fertilidad del suelo,altitud y pendiente)se evaluaron mediante un análisis de componentes de principales (ACP) yel coeficiente de correlación por rangos de Spearman. La relación entre composición florística y condiciones edáficas se evaluó mediante un análisis de correspondencia canónica (ACC) con las especies que presentaban más de dos individuosy con las 50 especies más abundantes; para estos análisis los datos edáficos se transformaron logarítmicamente.

Para determinar el grado en que el espacio, el suelo y la topografía explican la variación florística arbórea, se realizó la partición de la varianza de las abundancias de las especiescon respecto alespacio (polinomios $x, y, x y, x^{2}, y^{2}, x^{2} y, x y^{2}, x^{3} y y^{3}$ ) y al ambiente (topografía, suelos y no explicadas); este procedimiento se realizó empleando los valores de los eigenvaluecalculados en los ACC (BORCARD et al., 1992).Para ello, se realizó inicialmente un análisis de correspondencia simple (AC) de especies sin incluir datos ambientales; luego se corrieron separadamente ACC de especies con las variables edáficas, espaciales ytopográficas, y posteriormente con todas las variables; y finalmente,para determinar el porcentaje de explicación de lavariación se empleó la ecuación 1 (ANDERSON; GRIBBLE,1998).

Porcentaje de variación $=$ Total de valor de los sigenvalue del ACC $* 100$ Total del valor los sigenvalue del AC

\section{RESULTADOS}

\subsection{Caracterización de la diversidad,composición de especies y sitio}

Los valores de riqueza de especies, abundancia de individuos, diversidad y equidad registrados en la parcela E fueron ligeramente superiores a los de la parcela $U$, pero tales diferencias no fueron
significativas.Similarmente,la dominancia fue levemente menor en la parcela E, pero no significativa(Tabla 1).Las familias botánicas mejor representadas en términos de abundancia de individuos fueron Sapotaceae, Euphorbiaceae, Lecythidaceae, Arecaceae, Myristicaceae, Vochysiaceae, y Clusiaceae; mientras que las menosabundantes fueron Menispermaceae, Lacistemataceae, Aquifoliaceae, Sabiaceae, y Lissocarceae. En la Parcela E, las especies más abundantes fueron Pouteria sp1 (35 individuos), Oenocarpus bataua(34), Eschweilera pittieri(29), Mabea chocoensis (24) y Croton jorgei (17). En ésta parcela, el $67 \%$ de las especies tuvieron uno o dos individuos (49.7\% y $17.3 \%$, respectivamente). En la parcela U las especies más abundantes fueron Mabea chocoensis(45 individuos), Pouteria sp1(23), Qualea lineata(22), Wettinia quinaria(21), Eschweilera integrifolia(20) y Oenocarpus bataua(17). En ésta parcela, el 60.8\%de las especies tuvieron unoo dos individuos (46.9\% y 13.9\%, respectivamente). En ambas parcelas los suelos fueron ácidos y muy pobres en nutrientes, con niveles de P, Ca, Mg y K muy bajos y muy baja capacidad de intercambio catiónico efectiva (CICE). La topografía fue similar en ambas parcelas (pendiente promedia de $24.5^{\circ}$ ), lo mismo que la altitud (Tabla 1).

\subsection{Relación entre las condiciones de sitioy la diversidad arbórea}

Según los resultados del ACP, cinco componentes presentaron eigenvalues significativos $(\geq 1.0)$ y explicaron el $81.7 \%$ de la variabilidad de los datos desitio y de diversidad, mientras que los dos primeros componentes explicaron el 56.9\%; en ellos se evidenció la asociación positiva de la mayoría de los índices de diversidad y riqueza de especies conel Al del suelo; y negativa con el pH y la pendiente. Ladominancia florística(índices de Simpson y BergerParker)estuvoasociada ala pendiente(Figura 1). La altitud, Ca, CICE, P y M.O. presentaron poca asociación lineal con las variables de diversidad.

El análisis de correlación por rangos de Spearmancorroboró los resultados anteriores, puesevidenció correlaciones positivas significativas de la riqueza,diversidad de Margalef y equidad de Shannon-Wiener con Al y negativas con la pendiente. Por su parte, la dominancia de Simpson presentó correlación negativacon $\mathrm{Al}$ y positiva con la pendiente; mientras que la dominancia de Berger-Parker correlacionó

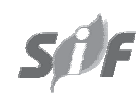

Revista Árvore, Viçosa-MG, v.38, n.6, p.1123-1132, 2014 
Tabela 1 - Diversidad de árboles y condiciones edáficas de dos parcelas en un bosque pluvial tropical del Chocó Biogeográfico, Colombia. Mín: valor mínimo, Máx: valor máximo, D.E.: desviación estándar, W: estadístico de la prueba de MannWhitney-Wilcoxon, Valor P: significancia estadística.

Tabela 1 - Diversidade de árvores e condições do solo de duas parcelas em uma floresta tropical de Chocó Biogeográfico, Colômbia. Min: valor mínimo, Max: valor máximo, D.E.: desvio-padrão, W: teste estatístico de Mann-WhitneyWilcoxon e Valor P: significância estatística.

\begin{tabular}{|c|c|c|c|c|c|c|c|c|c|c|c|c|}
\hline \multirow[t]{2}{*}{$\begin{array}{c}\text { Índices de } \\
\text { diversidad Alfa }\end{array}$} & \multicolumn{5}{|c|}{ Parcela E } & \multicolumn{5}{|c|}{ Parcela U } & \multicolumn{2}{|c|}{$\begin{array}{l}\text { Mann-Whitney } \\
\text {-Wilcoxon }\end{array}$} \\
\hline & Total & Media & Mín. & Máx. & D.E. & Total & Media & Mín. & Máx. & D.E. & $\mathrm{W}$ & Valor P \\
\hline Riqueza & 223 & 22.76 & 12 & 32 & 5.3 & 174 & 21.56 & 12 & 30 & 4.8 & -52.5 & 0.31 \\
\hline Abundancia & 716 & 28.48 & 16 & 43 & 7.26 & 640 & 25.76 & 13 & 34 & 5.44 & -65 & 0.21 \\
\hline Dominancia de Simpson & 0.01 & 0.06 & 0.04 & 0.11 & 0.02 & 0.02 & 0.06 & 0.04 & 0.09 & 0.02 & 21.5 & 0.68 \\
\hline $\begin{array}{l}\text { Equidad de Shannon- } \\
\text { Wiener }\end{array}$ & 4.81 & 3.01 & 2.39 & 3.37 & 0.26 & 4.63 & 2.98 & 2.46 & 3.36 & 0.25 & -35.5 & 0.5 \\
\hline Diversidad de Margalef & 33.19 & 6.49 & 3.97 & 8.37 & 1.19 & 27.52 & 6.32 & 4.29 & 8.22 & 1.12 & -32.5 & 0.53 \\
\hline Diversidad Alfa de Fisher & 108.1 & 74.91 & 18.15 & 177.6 & 47.1 & 82.13 & 89.98 & 20.98 & 346.6 & 68.74 & 45.5 & 0.38 \\
\hline $\begin{array}{l}\text { Dominancia de Berger- } \\
\text { Parker }\end{array}$ & 0.05 & 0.11 & 0.06 & 0.21 & 0.03 & 0.07 & 0.11 & 0.06 & 0.21 & 0.04 & -11 & 0.84 \\
\hline Diversidad Beta & \multicolumn{5}{|c|}{ Parcela E } & & \multicolumn{3}{|c|}{ Parcela U } & & \multicolumn{2}{|c|}{ Total } \\
\hline Familias Botánicas & \multicolumn{6}{|c|}{47} & \multicolumn{4}{|c|}{46} & \multicolumn{2}{|c|}{53} \\
\hline $\begin{array}{l}\text { Especies exclusivas } \\
\text { (porcentaje) }\end{array}$ & \multicolumn{6}{|c|}{127 (56.9\%) } & \multicolumn{4}{|c|}{$78(44.82 \%)$} & \multicolumn{2}{|c|}{$205(68.1 \%)$} \\
\hline $\begin{array}{l}\text { Especies compartidas } \\
\text { (porcentaje) }\end{array}$ & \multicolumn{6}{|c|}{$96(43.03 \%)$} & \multicolumn{4}{|c|}{$96(55.17 \%)$} & \multicolumn{2}{|c|}{96 (31.89\%) } \\
\hline Especies/individuos & \multicolumn{6}{|c|}{0.3114} & \multicolumn{4}{|c|}{0.2718} & \multicolumn{2}{|c|}{0.2219} \\
\hline Abundancia de individuos & \multicolumn{5}{|c|}{716} & & \multicolumn{3}{|c|}{640} & & & 356 \\
\hline \multirow[t]{2}{*}{ Variables Edáficas } & \multicolumn{6}{|c|}{ Parcela E } & \multicolumn{6}{|c|}{ Parcela U } \\
\hline & \multicolumn{3}{|c|}{ Media \pm D.E. } & \multicolumn{3}{|c|}{ Rango } & \multicolumn{3}{|c|}{ Media \pm D.E. } & & \multicolumn{2}{|c|}{ Rango } \\
\hline$\overline{\mathrm{pH}}$ & \multicolumn{3}{|c|}{$4.69 \pm 0.13$} & \multicolumn{3}{|c|}{$4.46-4.96$} & & $4.84 \pm$ & 0.15 & & $4.54-$ & -5.19 \\
\hline Materia orgánica (\%) & 5.64 & \pm 2.04 & & & $2-9$ & & & $7.87 \pm$ & 1.98 & & $4.18-$ & -10.14 \\
\hline Fósforo (ppm) & 1.97 & \pm 0.56 & & & $6-3$ & & & $1.31 \pm$ & 0.49 & & $0.7-$ & -3.01 \\
\hline Aluminio (meq/100g) & 0.49 & \pm 0.07 & & & $4-0$ & & & $0.47 \pm$ & 0.11 & & $0.3-$ & -0.8 \\
\hline Calcio (meq/100g) & 0.30 & \pm 0.15 & & 0.18 & $9-0$ & 951 & & $0.34 \pm$ & 0.16 & & 0.147 & -0.787 \\
\hline Magnesio (meq/100g) & 0.24 & \pm 0.12 & & 0.13 & $5-0$. & 549 & & $0.29 \pm$ & 0.12 & & 0.145 & -0.643 \\
\hline Potasio (meq/100g) & 0.23 & \pm 0.06 & & 0.08 & $3-0$ & 362 & & $0.21 \pm$ & 0.06 & & 0.097 & -0.344 \\
\hline $\begin{array}{l}\text { Topografía ( pendiente } \\
\text { en grados) }\end{array}$ & 24.54 & \pm 14.1 & & & $0-56$ & & & $24.52 \pm$ & 15.68 & & $2.0-$ & -51.5 \\
\hline Altitud (msnm) & 137.20 & $0 \pm 19$ & & 117 & $0-18$ & 0.0 & & $46.44 \pm$ & 18.68 & & 110.0 & -174.0 \\
\hline
\end{tabular}

negativamenteconK.La abundancia de individuos presentó correlación negativa débil pero significativa con la M.O., pH y topografía (Tabla 2).

\subsection{Relación entre las condiciones de sitioy la composición florística arbórea}

En el ACC para todas las especies arbóreas y las condiciones de sitio (edáficas, topográficas y espaciales) los cinco primeros ejes fueron significativos y explicaron el 77\% de la variación total de las especies.En el ACC para las 50 especies más abundantes se encontró que los dos primeros ejes explicaron el 50.7\% de la variación de la composición de especies con respecto al sitio.
El primer eje presentó un eigenvalue de 0.23 y explicó el 30.7\% de la variación, mientras que el segundo presentó un eigenvalue de 0.15 y explicó el restante $20 \%$; ambos fueron significativos $(P<0.05)$.

El ACC de las 50 especies dominantes mostró asociación entre las especies Qualea sp1, Chrysophyllum sp1, Hortia columbiana, Guarea glabra, Eschweilera pittieri, Matisia bullata, Pouteriamultiflora, Osteophloemplatyspermum,yEschweilera spcon el $\mathrm{P}$ del suelo. Las especies Licania macrocarpa, Dussia sp, Virola flexuosa, Wettiniaquinaria, yPouteria sp., presentaron asociación con laM.O.; así mismo, Minquartiaguianensis, Iryantheramegistophylla, 


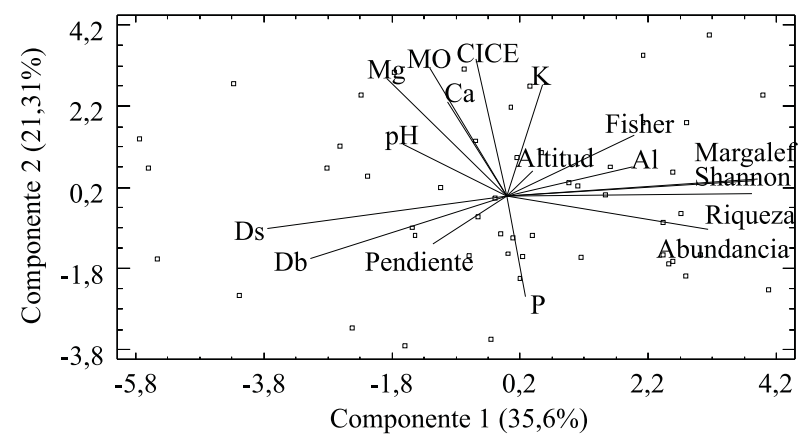

Figura 1 - Análisis de Componentes Principales (ACP) para variables de sitio (Al, Ca, Mg, K, P, CICE, pH, MO, Altitud y Pendiente) y de diversidad arbórea (Margalef, Shanon, Fisher, Dominancia de Simpson -Ds-, Dominancia de Berger Parker -Db-, Riqueza y Abundancia) de un bosque pluvial tropical del Chocó biogeográfico, Colombia.

Figura 1 - Análise de Componentes Principais das variáveis do sítio (Al, Ca, Mg, K, P, CICE, $p H, M O$,Altitudy Pendiente) e da diversidade de árvores (Margalef, Shanon, Fisher, Dominancia de Simpson-Ds-, Dominancia de Berger Parker-Db-, Riqueza y Abundancia) em uma floresta tropical de Chocó, Colômbia.
Guatteriaamplifolia, Otobalehmannii, Welfiageorgii, Trichiliapoeppiggi, Pouroumachocoana, Protium apiculatumestuvieron asociadas con la topografía, $\mathrm{Ca}, \mathrm{Mg}$ y K. Por otra parte, las especies Clusia sp2, Chrysophyllum sp, Qualealineata, Licaniadurifolia, yMatisiavaldes-bermejoi se asociaron con la Altitud; y las especies Eschweileraaff.integrifolia, Cassipoureasp, Sterculiaaerisperma, Xylopiacolumbiana, Mabeachocoensis, Vochysiasp, yAspidospermamegalocarpum se asociaron con el pH.Las especies Brosimumguianensis, Couratariguianensis, Pausandraaff.Martinii, Roucheriacf.monsalveae, Ingacocleensis, Gustaviasuperba, Ardisiacogolloi, Dacryodesoccidentalis, Cespedeziamacrophylla, Iryantheraulei, Crotonjorgei, Oenocarpusbataua, Pouteriasp1, Vochysiaferruginea y Brosimum utileno presentaron asociación significativa con las variables edáficas. El contenido de Al presentó poca relación con las especies (Figura 2).

La partición de la variación total de la composición florística de las especies arbóreas mostró que las

Tabela 2 - Coeficientes de correlación por rangos de Spearman entre las variables edáficas y de diversidad arbórea de dos parcelas de un bosque pluvial tropical del Chocó, Colombia. Valor P: significancia estadística de la correlación. Los cuadros en gris muestran correlaciones significativas. $\mathrm{N}=50$.

Tabela 2 - Coeficientes de correlação de Spearman entre as variáveis de solo e diversidade de árvores de duas parcelas de uma floresta tropical de Chocó, Colômbia. Valor P: significância estatística da correlação. As caixas em cinza mostram correlações significativas. $N=50$.

\begin{tabular}{|c|c|c|c|c|c|c|c|}
\hline Diversidad & Abundancia & Riqueza & Margalef & $\begin{array}{c}\text { Shannon } \\
\text { Wiener }\end{array}$ & Alfa Fisher & Simpson & $\begin{array}{l}\text { Berger- } \\
\text { Parker } \\
\end{array}$ \\
\hline \multicolumn{8}{|l|}{ Edáficas } \\
\hline Altitud & 0.1599 & 0.0510 & 0.0011 & -0.0046 & -0.1634 & 0.0637 & 0.0710 \\
\hline Valor $P$ & 0.2631 & 0.7212 & 0.9937 & 0.9740 & 0.2526 & 0.6557 & 0.6193 \\
\hline Aluminio & 0.2312 & 0.3144 & 0.3423 & 0.3406 & 0.1743 & -0.3149 & -0.2287 \\
\hline Valor $P$ & 0.1056 & 0.0278 & 0.0166 & 0.0171 & 0.2223 & 0.0275 & 0.1094 \\
\hline Calcio & -0.1048 & -0.0212 & -0.0059 & 0.0018 & 0.0303 & -0.0090 & -0.0933 \\
\hline Valor $P$ & 0.4630 & 0.8821 & 0.9670 & 0.9898 & 0.8322 & 0.9496 & 0.5135 \\
\hline CICE & -0.1482 & -0.0469 & -0.0008 & 0.0149 & 0.0628 & -0.0447 & -0.1752 \\
\hline Valor $P$ & 0.2995 & 0.7427 & 0.9957 & 0.9167 & 0.6601 & 0.7546 & 0.2201 \\
\hline Fósforo & 0.1884 & 0.0870 & -0.0026 & -0.0081 & -0.1789 & 0.0556 & 0.2004 \\
\hline Valor $P$ & 0.1873 & 0.5426 & 0.9856 & 0.9546 & 0.2104 & 0.6974 & 0.1606 \\
\hline Magnesio & -0.2658 & -0.2195 & -0.1841 & -0.1725 & -0.0043 & 0.1326 & -0.0398 \\
\hline Valor $P$ & 0.0628 & 0.1244 & 0.1976 & 0.2272 & 0.9759 & 0.3534 & 0.7805 \\
\hline M.O.\%. & -0.2689 & -0.2137 & -0.1472 & -0.1398 & 0.1115 & 0.0889 & -0.0744 \\
\hline Valor $P$ & 0.0598 & 0.1347 & 0.3028 & 0.3279 & 0.4352 & 0.5337 & 0.6027 \\
\hline $\mathbf{p H}$ & -0.2797 & -0.2203 & -0.1882 & -0.1890 & 0.0970 & 0.1488 & 0.0388 \\
\hline Valor $P$ & 0.0503 & 0.1230 & 0.1878 & 0.1858 & 0.4970 & 0.2976 & 0.7859 \\
\hline Potasio & 0.0874 & 0.1490 & 0.1772 & 0.2023 & 0.1131 & -0.2214 & -0.3167 \\
\hline Valor $P$ & 0.5405 & 0.2971 & 0.2147 & 0.1568 & 0.4287 & 0.1213 & 0.0266 \\
\hline Pendiente & -0.2842 & -0.3552 & -0.3609 & -0.3635 & -0.2570 & 0.3147 & 0.1680 \\
\hline Valor $P$ & 0.0466 & 0.0129 & 0.0115 & 0.0109 & 0.0720 & 0.0276 & 0.2396 \\
\hline
\end{tabular}


Códigos de las especies: 1 Mabea chocoensis, 2 Pouteria sp1, 3 Oenocarpus bataua, 4 Eschweilera pittieri, 5 Croton jorgei, 6 Wettinia quinaria, 7 Eschweilera aff. integrifolia, 8 Brosimum utile, 9 Qualea lineata, 10 Matisia bullata, 11 Welfia georgii, 12 Pouteria multiflora, 13 Otoba lehmannii, 14 Eschweilera sp, 15 Chrysophyllum sp, 16 Iryanthera ulei, 17 Gustavia superba, 18 Iryanthera megistophylla, 19 Cassipourea sp, 20 Minquartia guianensis, 21 Pausandra aff. Martinii, 22 Pouteria sp2, 23 Pouteria sp3, 24 Brosimum guianensis, 25 Couratari guianensis, 26 Dacryodes occidentalis, 27 Pourouma chocoana, 28 Xylopia columbiana, 29 Aspidosperma aff. Megalocarpum, 30 Chrysophyllum sp1, 31 Roucheria cf. Monsalveae, 32 Qualea sp1, 33 Sterculia aerisperma, 34 Vochysia ferruginea, 35 Ardisia cogolloi, 36 Guarea glabra, 37 Guatteria aff. Amplifolia, 38 Hortia Columbiana, 39 Protium apiculatum, 40 Virola flexuosa, 41 Inga cocleensis, 42 Licania durifolia, 43 Licania macrocarpa, 44 Matisia valdes-bermejoi, 45 Osteophloem platyspermum, 46 Trichilia poeppiggi, 47 Vochysia sp, 48 Cespedezia macrophylla, 49 Clusia sp2, 50 Dussia sp.

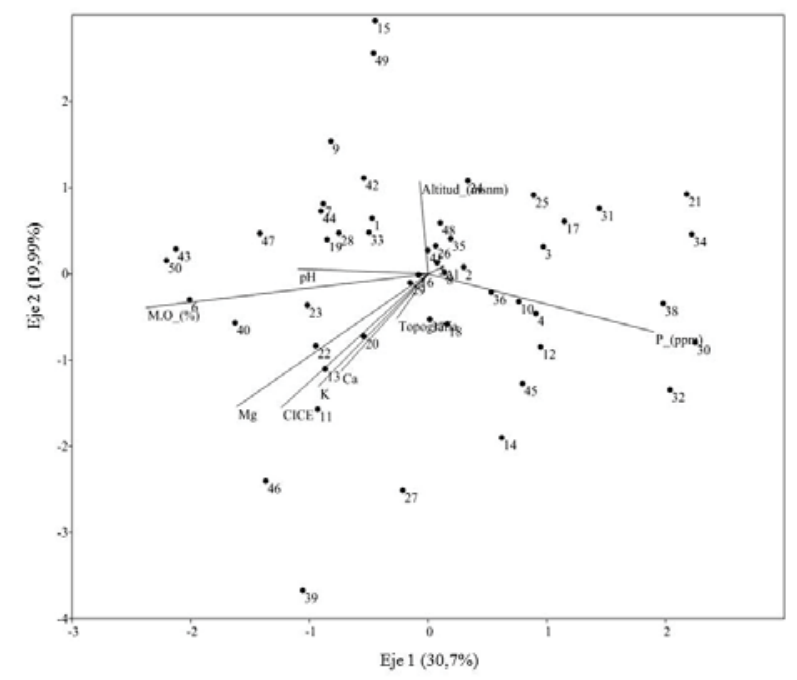

Figura - 2 Análisis de Correspondencia Canónica (ACC) para variables de sitio y la composición florística arbórea de las 50 especies más abundantes deun bosque pluvial tropical del Chocó biogeográfico, Colombia.

Figura 2 - Análise de correspondência canônica (ACC) das variáveis do site e composição florística arbórea das 50 espécies mais abundantes em uma floresta tropical de Chocó, Colômbia.

variables topográficas (pendiente y altitud) explicaron el $4.1 \%$ de la variación, las edáficas explicaron el 15.8\%, los polinomios espaciales el $17.3 \%$ y el restante $62.6 \%$ quedó sin explicar (Figura 3). De manera similar,la partición de la variación total de la composición florística de las 50 especies arbóreas más abundantes mostró que las variables topográficas explicaron el 4.4\% de la variación, las edáficas el 17\%, las espaciales el 20.5\%, mientras queel 57.91\% de la variación quedó sin explicar (Figura 3).

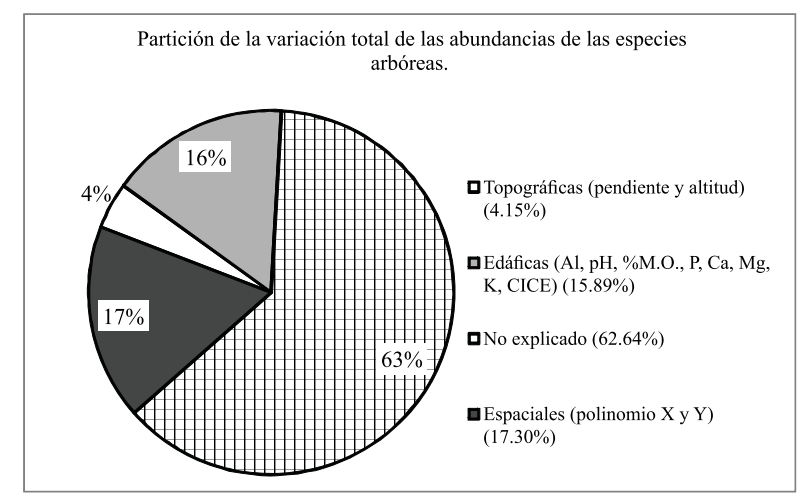

Partición de la variación total de las abundancias de las 50 especies arbóreas más dominantes.

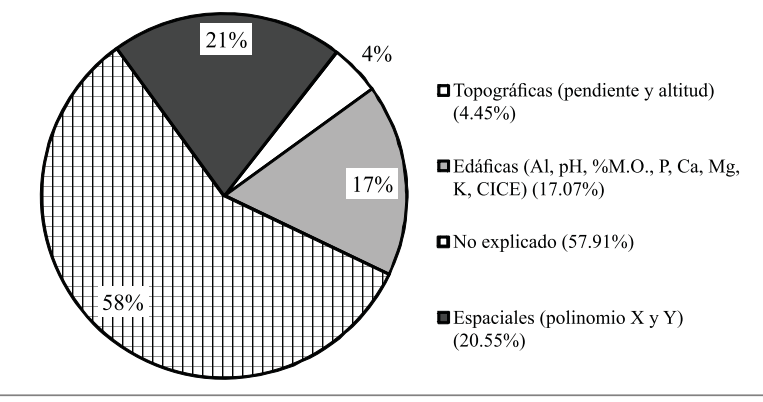

Figura 3 - Partición de la varianza de las abundancias de las especies arbóreas (total y 50 especies más dominantes) y las variables de sitio (topográficas, edáficas, espaciales y no explicadas) de un bosque pluvial tropical del Chocó Biogeográfico, Colombia.

Figura 3-Partição da variância das abundâncias das espécies arbóreas (total e 50 espécies mais dominantes) e variáveis do site (topográficas, do solo, espaciais e sem explicação) de uma floresta tropical de Chocó, Colômbia.

\section{DISCUSIÓN}

\section{1. ¿Cómo están distribuidas las abundancias de las especies arbóreas en un bosque pluvial tropical del Chocó Biogeográfico?}

Se evidenció que la mayoría de las especies están representadas por un número reducido de individuos, generalmente uno. Dominan unas pocas especies y familias botánicas, las cualesgeneralmente agrupan más del 50\% de la abundancia de individuos, lo cual ha sido ampliamente reportado en bosques húmedos tropicales del mundo(HUBBELL, 1997;TER STEEGE et al., 2013).La alta abundancia de la familia Arecaceaeencontrada en el presente estudio, concuerda con lo reportado en esta 
región por Gentry (1993b), Galeano(2000), Ramírez; Galeano (2011) y Nieto (2009). Particularmente, Oenocarpus bataua presentó alta abundancia en ambas parcelas y ha sido reportada como una de las especies dominantesen estos ecosistemas (FABER-LANGENDOEN; GENTRY, 1991;RAMÍREZ; GALEANO, 2011;NIETO, 2009), lomismo que en bosques de distintas unidades fisiográficas de la Amazonia (TER STEEGE et al., 2013).

La especie de mayor abundancia en el bosque del presente estudio(Mabea chocoensis) también ha sido reportada como una de las de mayor importancia ecológicaen otros bosques secundarios de la misma región del Chocó biogeográfico(NIETO, 2009).Demanera similar, las familias más abundantes del presente estudio fueronSapotaceae, Euphorbiaceae, Lecythidaceae, Arecaceae, y Myristicaceae, también han sido reportadascomo dominantes en otros bosques del Chocó(FABERLANGENDOEN; GENTRY, 1991;GENTRY, 1993b;GALEANO, 2000; NIETO, 2009).Estos resultados soportan la hipótesis de hiperdominancia de unos pocos grupos biológicos en los bosques húmedos tropicales; así mismo, es notorio que estudios recientes realizados en la Amazonia (PITMAN et al.,1999, TER STEEGE et al., 2013),también han registrado la hiperdominancia de algunas de las familiasencontradas en el presente estudio (como Lecythidaceae, Arecaceae, y Myristicaceae).Esta coincidencia de resultados en dos regiones geográficas tan distantes se puede explicar porque las especies hiperdominantes tienen rangos de distribución más grandes que los otros taxa. Sin embargo, la mayoría de tales especies no son generalistas como cabría esperarse por su amplia distribución, sino que son especialistas de hábitat, pues muestran mayor preferencia por un tipo debosque específico. Aunque no están claras las causas de las altas densidades locales de las especies hiperdominantes, se ha propuesto que tales especies deben presentar alta resistencia a patógenos, herbívoros especialistas y otras causas de mortalidad asociadas a la denso dependencia (TER STEEGE et al., 2013).

\section{2. ¿Existe relación entre la composición florística, los patrones de riqueza, diversidad y dominancia florística arbórea con las condiciones edáficas en este bosque?}

Las correlaciones significativas positivas, aunque débiles, entre diversos índices de diversidad y riqueza de especies con el Al del suelo encontrados acá (Tabla 2), también han sido reportados en estudios realizados en distintos bosques de la Amazonía (LAURANCE et al., 2010; BARRETO et al., 2010; CALLE et al., 2011). Estas tendencias se deben probablemente al efecto de la toxicidad del Al sobre las plantas y específicamente sus efectos deletéreos sobre el crecimiento y función de las raíces (SOLLINS, 1998); esta toxicidad, probablemente induce mayor mortalidad y disminuye el crecimiento, lo cual reduciría la dominancia e incrementaría la diversidad de árboles pequeños.

La disminución de la abundancia, riqueza y diversidad de especies con el incremento en la pendiente (Tabla 2),probablemente se debe a condiciones más adversas para el establecimiento de los árbolesen las zonas de mayor inclinación, lo cual genera mayor dominancia y reducción en diversidad florística.Estos resultados, concuerdan conlos hallazgos de otros estudios realizados en bosques húmedos tropicales, en los cuales se ha documentado la influencia de la topografía sobre la diversidad y composición florística arbórea (VALENCIA et al., 2004; BARRETO et al., 2010; LAURANCE et al., 2010). Por ejemplo, a escala local, las condiciones edáficas explicaron la variación de algunas especies dominantes del bosque,las cuales se pueden catalogar como especialistas de hábitat.Entre ellas,Qualea sp1, Chrysophyllumsp1, Hortiacolumbiana, que se asociaron alaconcentración de $\mathrm{P}$ del suelo y Licaniamacrocarpa, Dussiasp, Virolaflexuosa, Wettiniaquinariaque se asociaron al contenido de M.O.

Pero dado que las variables espaciales y ambientales (topográficas y edáficas) explicaron solamente un 37\% de la variación de la composición florística de todas las especies y un $42 \%$ de tal variación de las 50 especies dominantes (Figura 3), parece serque la distribución de las especiesa escala local está gobernada principalmentepor procesos aleatorios o biológicos (como la limitación en dispersión), conforme lo propone la teoría neutral (HUBBELL,2001;HE; HU, 2005). Según esta teoría,las probabilidades de nacimiento, muerte, migración y especiación de todos los organismos en una comunidad no están afectadas por presión de selección, y en consecuencia, la abundancia de una especie en una comunidad no responde al efecto acumulado de eventos tales como interacciones entre las especies o condiciones heterógeneas de hábitat. Nuestros resultados sugieren que el efecto de las variables espaciales y edáficas sobre la diversidad y composición florística eslimitado, lo cual se ha

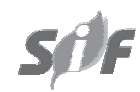

Revista Árvore, Viçosa-MG, v.38, n.6, p.1123-1132, 2014 
evidenciado en otros bosques húmedos tropicales (DUQUE et al., 2003; HE et al., 2002;VALENCIA et al., 2004; BARRETO et al.,2010)

De acuerdo con lo anterior, el papel de las adaptaciones específicas al hábitat (teoría del nicho) sobre la diversidad y composición florística (HUTCHINSON, 1959; TILMAN; PACALA, 1993) pareciera ser limitado cuando el análisis se hace a escala local. No obstante, como se mencionó atrás, los análisis de hiperdominancia realizados en grandes paisajes, sugieren que tales especies son especialistas de hábitat (TER STEEGE et al., 2013), lo cual también ocurrió con algunas de nuestras especies dominantes, cuya variación fue explicada por las características edáficas, según se mostró atrás. En síntesis, los resultados del presente estudioconcuerdan con numerosos trabajos que han concluido que la diversidad y distribución de especies arbóreas de bosques tropicales se encuentran determinadas parcialmente por variables ambientales como fertilidad y tipo del suelo, topografía, drenaje, régimen de inundación y espacio, así como por procesos aleatorios o biológicos,entre otros, los cuales operan a diferentes escalas (regional y local) (TUOMISTO et al., 2003; JONES et al., 2008; LAURANCE et al., 2010; BARRETO et al., 2010).

\section{CONCLUSIONES}

Los resultados soportan la hipótesis de hiperdominancia de unos pocos grupos biológicos en bosques húmedos tropicales. La topografía y el Al del suelo explicaron en partela diversidad florística. No obstante,la distribución de las especies a escala local parece estar gobernada principalmente por procesos aleatorios o biológicos (como la limitación en dispersión), conforme a la teoría neutral; el papel de las adaptaciones específicas al hábitat (teoría del nicho) pareciera ser limitado. En síntesis, la diversidad y distribución de las especies arbóreas de los bosques estudiados parecen estar determinadas parcialmente por variables ambientales comotopografía, fertilidad y tipo del suelo, entre otros, así como por procesos aleatorios o biológicos,los cuales probablemente operan a diferentes escalas espaciales y temporales.

\section{RECONOCIMIENTOS}

Este trabajo fue cofinanciado por la Empresa Interconexión Eléctrica S. A.(I.S.A.)mediante el programa de becas de investigación I.S.A. - Universidad Nacional
2008 y por la Universidad Nacional de Colombia,Sede Medellín.

\section{REFERENCIAS}

ANDERSON, M.; GRIBBLE, N. Partitioning the variation among spatial, temporal and environmental components in a multivariate data set. Australian Journal of Ecology, v.23, n.1, p.158-167, 1998.

ANTONELLI, A.; SANMARTIN, I. Why are there so many plant species in the Neotropics?.

Taxon, v.60, n.2, p.403-414, 2011.

BARRETO, J. S.; MONTOYA, A. J. D.; LOPEZ, D. C.; HURTADO, F. H. M. Variación florística de especies arbóreas a escala local en un bosque de tierra firme en la Amazonia colombiana. Acta Amazónica, v.40, n.1, p.179-188, 2010.

BORCARD, D.; LEGENDRE, P.; DRAPEAU, P. Partialling out the spatial Component of Ecological Variation. Ecology, v.73, n.3, p.1045-1055, 1992.

CALLE, B.; MORENO, F.; CÁRDENAS, D. Relación entre suelos y estructura del bosque en la Amazonía colombiana. Revista de Biología Tropical, v.59, n.3, p.1307-1322, 2011.

DUIVENVOORDEN, J. F. Tree species composition and rain forest-environment relationships in the middle Caqueta, Colombia, NW Amazonia.

Vegetatio, v.120, n.1, p.91-113, 1995.

DUQUE, A.; CAVELIER, J.; POSADA, A. Strategies of tree occupation at a local scale in terra firme forests in the Colombian Amazon. Biotropica, v.35, v. 2, p.20-27, 2003.

FABER-LANGENDOEN, D.; GENTRY, A. The structure and diversity of rain forests at Bajo Calima, Chocó Region, Western Colombia.

Biotropica, v.23, n.1, p.2-11, 1991.

GALEANO, G. Forest use at the Pacific coast of Chocó, Colombia: a quantitative approach. Economic Botany, v.54, n.3, p.358-376, 2000.

GENTRY, A. Changes in plant community diversity and floristic composition on environmental and geographic gradients. Annals of the Missouri Botanical Garden, v.75, p.1-34, 1988. 
GENTRY, A. A field guide to the families and genera of woody plants of Northwest South American. Washington: Conservation International, 1993a. 895p.

GENTRY, A. Riqueza de especies y composición florística de las comunidades de plantas de la Región del Chocó: una actualización. em: LEYVA, P. (Ed). Colombia Pacífico. Santa Fe de Bogotá: Fondo para la Protección del Medio Ambiente FEN, 1993b. tomo 1. p.200-219.

HARTSHORN, G. S. Biogeografía de los bosques neotropicales. In GUARIGUATA, M. R.; KATTAN, H. (Ed). Ecología y conservación de bosques neotropicales. Cartago: LUR, 2002. p.59-81.

HE, F.; HU, X. S. Hubbell's fundamental biodiversity parameter and the Simpson diversity index. Ecology Letters, v.8, p.386-390, 2005.

HE, F.; LAFRANKIE, J.; SONG, B. Scale dependence of tree abundance and richness in a tropical rain forest, Malaysia. Landscape Ecology, v.17. n.6, p.559-568, 2002.

HUBBELL, S. P. A uniûed theory of biogeography and relative species abundance and its application to tropical rain forests and coral reefs. Coral Reefs, v. 16. p.S9-S21, 1997.

HUBBELL, S. P. The unified neutral theory of biodiversity and biogeography.. Princeton University Press,. 2001. 375p.

(Monographs in Population Biology, 32)

HUTCHINSON, G. E. Homage to Santa Rosalia or why are there so many kinds of animals? The American Naturalist, v.93, n.1,p.145-159, 1959.

JOHN, R. DALLING, J.W.; HARMS, K.E.; YAVITT, J.B.; STALLARD, R.F.; MIRABELLO, M.; HUBBELL, S.P.; VALENCIA, R.; NAVARRETE, H.; VALLEJO, M.; FOSTER, R.B. Soil nutrients influence spatial distributions of tropical tree species. Proceedings of National Academy of Sciences, v.104, n.3, p.864-869, 2007.

JONES, M.; TUOMISTO, H.; BORCARD, D.; LEGENDRE, P.; CLARK, D.B.; OLIVAS, P.C.
Explaining variation in tropical plant community composition: influence of environmental and spatial data quality. Oecologia, v.155, n.3, p.593-604, 2008.

LAURANCE, S.; LAURANCE, W.F.; ANDRADE, A.; FEARNSIDE, P.M.; HARMS, K.E.; VICENTINI, A.; LUIZÃO R.C.C. Influence of soils and topography on Amazonian tree diversity: a landscape-scale study. Journal of Vegetation Science, v.21, n.1, p.96-106, 2010.

MAGURRAN, A. E. Ecological diversity and its measurement. Princeton: University Press, 1988. 179p.

MAHECHA, G. Fundamentos y metodologías para la identificación de plantas. Proyecto Biopacífico. . Bogotá: Ministerio del Medio Ambiente, GEF - PNUD, 1997. 282p.

MALAGÓN, D.; PULIDO, C.; LLINAS, R.D.; CHAMORRO, C.; FERNÁNDEZ, J. Suelos de Colombia. Origen, evolución, clasificación, distribución y uso. Santa fe de Bogotá: Instituto Geográfico Agustín Codazzi. Subdirección de Agrología, 1995. 632 p.

MYERS, N., MITTERMEIER, R. A.; MITTERMEIER, C.G.; DA FONSECA, G.A.B.; KENT, J. Biodiversity hotspots for conservation priorities. Nature, v.403, n.6772, p.853-858, 2000.

NIETO, A. Diversidad florística y elementos estructurales de los Bosques Secundarios del Bajo Calima. En: MELO, O.; LOZANO, L. (Ed). Los Bosques Secundarios del Trópico Húmedo Colombiano. Caso del bajo calima: características y expectativas para el manejo sostenible $y$ conservación de los ecosistemas boscosos del Bajo Calima. Buenaventura, Valle del Cauca, Colombia. Ibagué: Universidad del Tolima, 2009. p.41-55.

PHILLIPS, O.; HALL, R.; GENTRY, A.R.; SAWYER, S.A.; VIZQUEZ. R. Dynamics and species richness of tropical forests. Proceedings of the National Academy of Sciences. USA, v.91, n.7, p.2805-2809, 1994.

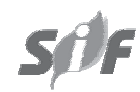

Revista Árvore, Viçosa-MG, v.38, n.6, p.1123-1132, 2014 
PITMAN, N.; TERBORGH, J.; SILMAN, M.R.; NUÑEZ, P. Tree species distributions in an upper Amazonian forest. Ecology, v.80, n.8, p.26512661, 1999.

POULSEN, A. D.; TUOMISTO, H.; BASLEV, H. Edaphic and floristic variation a 1-ha Plot of Lowland Amazonian Rain Forest. Biotropica, v.38, n.4, p.468-478, 2006.

RAMÍREZ, G.; GALEANO, G. Comunidades de palmas en dos bosques de Chocó, Colombia. Caldasia, v.33, n.2, p.315-329, 2011.

RANGEL, J. O. Colombia diversidad biótica IV: El Chocó biogeográfico. Costa Pacífica. Bogotá:.Instituto de ciencias Naturales Universidad Nacional de Colombia, 2004. 997p..

SOLLINS, P. Factors influencing species composition in tropical lowland rain forest: Does soil matter?. Ecology, v.79, n.1, p.23-30, 1998.

TER STEEGE, H.; JETTEN, V.; POLAK, A.; WERGER, M. Tropical rain forest types and soil factors in a watershed area in Guyana. Journal of Vegetation Science, v.4, n.5, p.705-716, 1993.

TER STEEGE, H.; PITMAN, N.C.A.; SABATIER, D.; BARALOTO, C.; SALOMÃO, R.P.; GUEVARA, J.E.; PHILLIPS, O.L.; CASTILHO, C.V.; MAGNUSSON, W.E.; MOLINO, J.; MONTEAGUDO, A.; NÚÑEZ VARGAS, P.; MONTERO, J.C.; FELDPAUSCH, T.R.; CORONADO, ENH.; KILLEEN, TJ.; MOSTACEDO, B.; VASQUEZ, R.; ASSIS, R L.; TERBORGH, J.; WITTMANN, F.; ANDRADE, A.; LAURANCE, W F.; LAURANCE, SG.; W.; MARIMON, B S.; MARIMON JR.;, GUIMARÃES VIEIRA, IC.; AMARAL, I L.; BRIENEN, R.; CASTELLANOS, H.; CÁRDENAS LÓPEZ, D.; DUIVENVOORDEN, J F.; MOGOLLÓN, H F.; DIONÍZIA DE ALMEIDA MATOS, F.; DÁVILA, N.; GARCÍA-VILLACORTA, R.; STEVENSON DIAZ, P R.; COSTA, F.; EMILIO, T.; LEVIS, C.; SCHIETTI, J.; SOUZA, P.; ALONSO, A.; DALLMEIER, F.; DUQUE, A J.; FERNANDEZ, $M$ T.; ARAUJO-MURAKAMI, A.; ARROYO, L.; GRIBEL, R.; FINE, P V.; A.; PERES, CA.; TOLEDO,
M.; AYMARD C.; G A.; BAKER, T R.; CERÓN, C.; ENGEL, J.; HENKEL, T W.; MAAS, P.; PETRONELLI, P.; STROPP, J.; ZARTMAN, C E.; DALY, D.; NEILL, D.; SILVEIRA, M.; RÍOS PAREDES, M.; CHAVE, J.; LIMA FILHO, D.; MØLLER JØRGENSEN, P.; FUENTES, A.; SCHÖNGART, J.; CORNEJO VALVERDE, F.; DI FIORE, A.; JIMENEZ, E M.; PEÑUELA, M C.; PHILLIPS, J F.; RIVAS, G.; VAN ANDEL, T R.; VON HILDEBRAND, P.; HOFFMAN, B.; ZENT, E L.; MALHI, Y.; PRIETO, A.; RUDAS, A.; RUSCHELL, A R.; SILVA, N.; VOS, V.; ZENT, S.; OLIVEIRA, AA.; CANO SCHUTZ, A.; GONZALES, T.; NASCIMENTO, MT.; RAMIREZ-ANGULO, H.; SIERRA, R.; TIRADO, M.; UMAÑA MEDINA, M N.; VAN DER HEIJDEN, G.; VELA, C I.; A.; VILANOVATORRE, E.; VRIESENDORP, C.; WANG, O.; YOUNG, K R.; BAIDER, C.; BALSLEV, H.; FERREIRA, C.; MESONES, I.; TORRES-LEZAMA, A.; URREGO GIRALDO, L E.; ZAGT, R.; ALEXIADES, M N.; HERNANDEZ, L.; HUAMANTUPA-CHUQUIMACO, I.; MILLIKEN, W.; PALACIOS CUENCA, W.; PAULETTO, D.; VALDERRAMASANDOVAL, E.; VALENZUELA GAMARRA, L.; DEXTER, K G.; FEELEY, K.; LOPEZGONZALEZ, G.; SILMAN, M R.; Hyperdominance in the Amazonian Tree Flora. Science, v.342. p.1243092. 2013.

TILMAN, D.; PACALA, S. The maintenance of species diversity in plant communities. Species diversity in ecological communities. Chicago: University of Chicago Press, 1993. p.13-25.

TUOMISTO, H.; RUOKOLAINEN, K.; YLI HALLA, M. Dispersal, environment, and floristic variation of western amazonian forests. Science, v.299, p.241-244, 2003.

VALENCIA, R.; FOSTER, R.B.; VILLA, G.; CONDIT, R.; SVENNING, J.; HERNÁNDEZ, C.; ROMOLEROUX, K.; LOSOS, E.; MAGÅRD, E.; BALSLEV, H.Tree species distributions and local habitat variation in the Amazon: large forest plot in eastern Ecuador. Journal of Ecology, v.92, n.4, p.214-229, 2004. 